PROCEEDINGS OF THE

AMERICAN MATHEMATICAL SOCIETY

Volume 135, Number 4, April 2007, Pages 1115-1121

S 0002-9939(06)08682-5

Article electronically published on October 27, 2006

\title{
SHARP MARCHAUD AND CONVERSE INEQUALITIES IN ORLICZ SPACES
}

\author{
Z. DITZIAN AND A. V. PRYMAK
}

(Communicated by Jonathan M. Borwein)

\begin{abstract}
For $L_{p}$ spaces on $\mathbb{T}^{d}, \mathbb{R}^{d}$ and $S^{d-1}$, sharp versions of the classical Marchaud inequality are known. These results are extended here to Orlicz spaces (on $\mathbb{T}^{d}, \mathbb{R}^{d}$ and $S^{d-1}$ ) for which $M\left(u^{1 / q}\right)$ is convex for some $q, 1<$ $q \leq 2$, where $M(u)$ is the Orlicz function. Sharp converse inequalities for such spaces are deduced.
\end{abstract}

\section{INTRODUCTION}

The sharp Marchaud inequality

$$
\omega^{r}(f, t)_{p} \leq C t^{r}\left\{\int_{t}^{1} \frac{\omega^{r+1}(f, u)_{p}^{q}}{u^{r q+1}} d u+\|f\|_{p}^{q}\right\}, \quad q= \begin{cases}p, & 0<p \leq 2, \\ 2, & 2 \leq p<\infty, \\ 1, & p=\infty,\end{cases}
$$

was proved for some measures of smoothness $\omega^{r}(f, t)_{L_{p}(\mathcal{D})} \equiv \omega^{r}(f, t)_{p}$ and some domains $\mathcal{D}$ (see $\mathrm{Zy}$, Ti], Di, 88, To, $\mathrm{Ti}, 99$ and $\mathrm{Da}-\mathrm{Di}, 05)$. Such results are an improvement over the classical Marchaud inequality, that is, (1.1) for $1 \leq p<\infty$ and $q=1$, and it was shown in $\mathrm{Zy}$ that $q$ of (1.1) is best possible for $1 \leq p<\infty$. Using a result of the type (1.1), one usually obtains

$$
\omega^{r}(f, t)_{p} \leq C t^{r}\left\{\sum_{n \leq \frac{1}{t}} n^{r q-1} E_{n}(f)_{p}^{q}\right\}^{1 / q}
$$

with the appropriate best $n$-th degree approximation $E_{n}(f)_{p}$. We will show here that $L_{p}(\mathcal{D}), 1<p \leq 2$ and $2 \leq p<\infty$, can be replaced by Orlicz spaces with the Orlicz function $M(u)$ for which $M\left(u^{1 / p}\right)$ and $M\left(u^{1 / 2}\right)$ are convex respectively.

There are two methods of proof for inequalities like (1.1). The first is by using a Littlewood-Paley type inequality (used by [Zy, [Ti] and Da-Di, 05) and the second one uses the concept of power-type $q$ from the geometry of Banach spaces (see Di, 88 and Di, 99]). Each method has advantages, that is, enables one to

Received by the editors November 9, 2005.

2000 Mathematics Subject Classification. Primary 26A15, 26B99, 41A27; Secondary 41A63, $46 \mathrm{E} 30$.

Key words and phrases. Sharp Marchaud inequality, Orlicz spaces, power-type $q$.

The first author was supported by NSERC grant of Canada A4816.

This research was done while the second author visited University of Alberta in 2005; the visit was supported by the first author's NSERC grant of Canada A4816.

(C)2006 American Mathematical Society Reverts to public domain 28 years from publication 
obtain a more general result than the other in some direction. Here we use a result from the geometry of Banach spaces ( $\mathrm{Ma}-\mathrm{Tr}]$ ) to obtain an inequality of type (1.1) for Orlicz spaces for which $M\left(u^{1 / q}\right)$ is convex for some $q, 1<q \leq 2$, where $M(u)$ is the Orlicz function. As we do not know a Littlewood-Paley type inequality for these spaces, it is natural to opt for the alternative method.

In Section 2 we derive the ingredients needed, and as corollaries obtain the analogues of (1.1) for $\mathbb{T}^{d}, \mathbb{R}^{d}$ and $S^{d-1}$ with the appropriate measure of smoothness $\omega^{r}(f, t)_{B}$. In Section 3 we give the analogues of (1.2) with the corresponding concepts of best approximation and the required additional proofs either quoted or given directly.

\section{The Sharp Marchaud INEQUality}

Traditionally, a Banach space $B$ is said to be of power-type $q$ if

$$
\eta_{B}(\tau) \equiv \sup _{\|h\|_{B}=1,\|g\|_{B}=\tau}\left(\frac{\|h+g\|_{B}+\|h-g\|_{B}}{2}-1\right) \quad \text { satisfies } \quad \eta_{B}(\tau) \leq K \tau^{q} .
$$

In $\mathrm{Di}, 88$ the following alternative equivalent definition was introduced and used for proving the sharp Marchaud inequality:

$$
\frac{\|h+g\|_{B}+\|h-g\|_{B}}{2} \leq\left(\|h\|_{B}^{q}+L\|g\|_{B}^{q}\right)^{1 / q}
$$

where $L$ is independent of $h$ and $g$.

The Orlicz function $M(t)$ on $[0, \infty)$ is an increasing convex function satisfying $M(0)=0$. We assume here (as is commonly done) the $\Delta_{2}$ condition, that is, $M(2 t) \leq b M(t)$ for $t \in\left[0, t_{0}\right]$ and for $t \in\left[t_{1}, \infty\right)$. The Orlicz class of functions on $\mathcal{D}$ with a positive measure $d \mu(x)$ is the class of all functions $f$ for which

$$
\int_{\mathcal{D}} M(|f(x)|) d \mu(x)<\infty
$$

and the norm (in the Luxemburg form) is

$$
\|f\|_{X}=\inf \left\{\sigma>0: \int_{\mathcal{D}} M(|f(x)| / \sigma) d \mu(x) \leq 1\right\} .
$$

Maleev and Troyanski Ma-Tr, Theorem 1] proved the following theorem.

Theorem 2.1 (Maleev-Troyanski). Suppose that $M(t)$ is an Orlicz function satisfying the $\Delta_{2}$ condition and that $M(l t) \leq \frac{1}{2} l M(t)$ for some $l<1$. Then there exists an Orlicz function $N(t)$ equivalent to $M(t)$ such that

$$
\eta_{B}(\tau) \leq \tau^{2} \sup \left\{\frac{M(u v)}{u^{2} M(v)} ; u \in[\tau, 1], v \in(0, \infty)\right\},
$$

where $B$ is the Orlicz space generated by the Orlicz function $M(t)$ but endowed with the equivalent norm

$$
\|f\|_{B}=\inf \left\{\sigma>0: \int_{\mathcal{D}} N(|f(x)| / \sigma) d \mu(x) \leq 1\right\} .
$$

Lemma 2.2. Suppose $M\left(u^{1 / q}\right)$ is convex for some $q, 1<q \leq 2$, where $M(u)$ is an Orlicz function satisfying the $\Delta_{2}$ condition. Then there exists an Orlicz function $N(u), A^{-1} N(u) \leq M(u) \leq A N(u)$ and $\eta_{B}(\tau) \leq c \tau^{q}$ for $\eta_{B}(\tau)$ given by (2.1) with the norm of $B$ given by $(2.3)^{\prime}$. 
Proof. As $M\left(u^{1 / q}\right)$ is convex and $M(0)=0$, we have

$$
\frac{M\left(u_{1}\right)}{u_{1}^{q}} \leq \frac{M\left(u_{2}\right)}{u_{2}^{q}} \quad \text { for } \quad 0<u_{1}<u_{2} .
$$

Hence, for $l<1$

$$
\frac{M(l t)}{l^{q} t^{q}} \leq \frac{M(t)}{t^{q}} \text { and } M(l t) \leq l^{q} M(t),
$$

and we may choose $l$ such that $l^{q}<\frac{1}{2} l$. We now use (2.4) to obtain

$$
\begin{aligned}
\eta_{B}(\tau) & \leq \tau^{2} \sup \left\{\frac{M(u v)}{u^{2} M(v)} ; u \in[\tau, 1], v \in(0, \infty)\right\} \\
& \leq \tau^{2} \sup \left\{\frac{(u v)^{q}}{u^{2} v^{q}} ; u \in[\tau, 1], v \in(0, \infty)\right\} \\
& =\tau^{2} \sup \left\{u^{q-2} ; u \in[\tau, 1]\right\}=\tau^{q} .
\end{aligned}
$$

Using Lemma 2.2, we obtain the sharp Marchaud inequality for $\mathbb{T}^{d}$ and $\mathbb{R}^{d}$.

Theorem 2.3. Suppose $B$ is an Orlicz space of functions on $\mathbb{T}^{d}$ or $\mathbb{R}^{d}$ with $M$ of Lemma 2.2, Then

$$
\omega^{r}(f, t)_{B} \leq C t^{r}\left\{\int_{t}^{1} \frac{\omega^{r+1}(f, u)_{B}^{q}}{u^{r q+1}} d u+\inf _{c \in \mathbb{R}}\|f-c\|_{B}^{q}\right\}^{1 / q},
$$

where

$\omega^{r}(f, t)_{B} \equiv \sup _{|h| \leq t}\left\|\Delta_{h}^{r} f\right\|_{B}, \quad \Delta_{h} f(x) \equiv f(x+h)-f(x), \quad \Delta_{h}^{r} f(x) \equiv \Delta_{h}\left(\Delta_{h}^{r-1} f(x)\right)$, and $\|f\|_{B}$ is any norm equivalent to (2.3).

The case $q=1$ in Theorems 2.3 and 2.4 was already covered by the classical Marchaud-type inequality (see also Di, 88 and Di, 99).

Proof of Theorem 2.3 . We use the norm given in $(2.3)^{\prime}$ by $N(u)$ and note that for this norm, translation is an isometry and $\eta_{B}(\tau) \leq c \tau^{q}$. Therefore, the conditions of Theorem 1 of Di, 88 are satisfied and (2.5) is valid with $\omega^{r}(f, t)_{B}$ defined by (2.6) using the norm $B$ given in $(2.3)^{\prime}$. Once (2.5) is satisfied for the norm given by $(2.3)^{\prime}$, it is valid for all equivalent norms (including that defined by (2.3)). We may write $f-c$ instead of $f$ as $\omega^{r}(f-c, t)_{B}=\omega^{r}(f, t)_{B}$ for $r \geq 1$.

Theorem 2.4. Suppose $B$ is an Orlicz space of functions on $S^{d-1}$ (the unit sphere in $\mathbb{R}^{d}$ ) with $M$ of Lemma 2.2. Then (2.5) is valid, where

$$
\begin{gathered}
\omega^{r}(f, t)_{B} \equiv \sup _{\rho \in O_{t}}\left\|\Delta_{\rho}^{r} f\right\|_{B}, \quad \Delta_{\rho} f(x) \equiv f(\rho x)-f(x), \quad \Delta_{\rho}^{r} f(x)=\Delta_{\rho}\left(\Delta_{\rho}^{r-1} f(x)\right), \\
O_{t} \equiv\left\{\rho \in \mathrm{SO}(d): \rho x \cdot x \geq \cos t \text { for all } x \in S^{d-1}\right\}
\end{gathered}
$$

where $\mathrm{SO}(d)$ is the group of $d \times d$ matrices of determinant 1 , and $B$ is given by any norm, equivalent to (2.3) with $\mathcal{D} \equiv S^{d-1}$ and the measure on the sphere induced by the Lebesgue measure. 
Proof. We note that (2.7) is the modulus of smoothness introduced in Di, 99 and follow the proof of Theorem 2.3 to deduce Theorem 2.4 from Di, 99, Theorem 3.1].

\section{Converse theorems}

In this section we will give estimates of $\omega^{r}(f, t)_{B}$ in terms of best approximation for the Orlicz spaces described in Lemma 2.2. We note that the concepts of best approximation on $\mathbb{T}^{d}, \mathbb{R}^{d}$ and $S^{d-1}$ differ and will be defined separately in each case. In this section, $n$ is a positive integer.

Theorem 3.1. Suppose $B$ is an Orlicz space of functions on $\mathbb{T}^{d}$ given by $M(u)$ of Lemma 2.2 for some $q, 1<q \leq 2$. Then

$$
\omega^{r}(f, t)_{B} \leq C t^{r}\left\{\sum_{n \leq \frac{1}{t}} n^{r q-1} E_{n}(f)_{B}^{q}\right\}^{1 / q},
$$

where

$$
E_{n}(f)_{B}=\inf \left\{\left\|f-T_{n}\right\|_{B}: T_{n} \in \operatorname{span} e^{i x \cdot l},|l| \equiv\left|\left(l_{1}, \ldots, l_{d}\right)\right| \equiv \max _{1 \leq i \leq d}\left|l_{i}\right|<n\right\} .
$$

Proof. We first observe that for a trigonometric polynomial of degree $n$ (as in (3.2)) we have

and hence

$$
\left\|\left(\frac{\partial}{\partial \xi}\right)^{r} T_{n}\right\|_{L_{\infty}\left(\mathbb{T}^{d}\right)} \leq d^{r / 2} n^{r}\left\|T_{n}\right\|_{L_{\infty}\left(\mathbb{T}^{d}\right)}
$$

$$
\left\|\Delta_{h}^{r} T_{n}\right\|_{L_{\infty}\left(\mathbb{T}^{d}\right)} \leq d^{r / 2} h^{r} n^{r}\left\|T_{n}\right\|_{L_{\infty}\left(\mathbb{T}^{d}\right)} .
$$

Therefore, for any Banach space $B$ of functions on $\mathbb{T}^{d}$ for which translation is an isometry

$$
\left\|\Delta_{h}^{r} T_{n}\right\|_{B} \leq d^{r / 2} h^{r} n^{r}\left\|T_{n}\right\|_{B} .
$$

For the proof of (3.4) (which follows from the technique in $\mathrm{Di}, 80$ ), we observe that for $g \in B^{*}$ (where $B^{*}$ is the dual to $B$ ), $T_{n} * g(u) \equiv\left\langle T_{n}(\cdot+u), g(\cdot)\right\rangle$ is a trigonometric polynomial that satisfies $\left\|T_{n} * g\right\|_{L_{\infty}\left(\mathbb{T}^{d}\right)} \leq\left\|T_{n}\right\|_{B} \cdot\|g\|_{B^{*}}$. We choose $g_{\varepsilon} \in B^{*},\left\|g_{\varepsilon}\right\|_{B^{*}}=1$ and set $Q_{n, \varepsilon}=T_{n} * g_{\varepsilon} \in L_{\infty}$ such that

$$
\begin{aligned}
\left\|\Delta_{h}^{r} T_{n}\right\|_{B}-\varepsilon & \leq\left|\Delta_{h}^{r}\left(T_{n} * g_{\varepsilon}\right)(0)\right|=\left|\Delta_{h}^{r}\left(Q_{n, \varepsilon}(0)\right)\right| \\
& \leq\left\|\Delta_{h}^{r} Q_{n, \varepsilon}\right\|_{L_{\infty}\left(\mathbb{T}^{d}\right)} \leq d^{r / 2} h^{r} n^{r}\left\|Q_{n, \varepsilon}\right\|_{L_{\infty}\left(\mathbb{T}^{d}\right)} \\
& \leq d^{r / 2} h^{r} n^{r}\left\|T_{n}\right\|_{B},
\end{aligned}
$$

and as $\varepsilon>0$ is arbitrary, (3.4) is established.

We observe that it is sufficient to prove (3.1) for some equivalent norm, and we use the Luxemburg form of the Orlicz space with $M(u)$ (or $N(u)$ of the MaleevTroyanski Theorem) for which translation is an isometry. We choose $c$ in Theorem 2.3 so that $E_{1}(f)_{B}=\|f-c\|_{B}$, and use the following (equivalent) form of (2.5):

$$
\omega^{r}\left(f, 2^{-n}\right)_{B} \leq C 2^{-n r}\left(\sum_{k=1}^{n} 2^{k r q} \omega^{r+1}\left(f, 2^{-k}\right)_{B}^{q}+E_{1}(f)_{B}^{q}\right)^{1 / q} .
$$


We set for the estimate of $\omega^{r+1}\left(f, 2^{-k}\right)_{B}$,

$$
f=f-P_{2^{k}}+\sum_{l=1}^{k}\left(P_{2^{l}}-P_{2^{l-1}}\right)+P_{1},
$$

where $P_{j}$, which is of degree $j-1$, satisfies $\left\|f-P_{j}\right\|_{B}=E_{j}(f)_{B}$. Using the above, we have

$$
\begin{aligned}
\omega^{r+1}\left(f, 2^{-k}\right)_{B} & \leq 2^{r+1} E_{2^{k}}(f)_{B}+2 d^{r / 2} \sum_{l=1}^{k} 2^{-k(r+1)} 2^{l(r+1)} E_{2^{l-1}}(f)_{B} \\
& \leq C_{1} \sum_{l=0}^{k} 2^{(l-k)(r+1)} E_{2^{l}}(f)_{B}
\end{aligned}
$$

Using Jensen's inequality, we derive

$$
\omega^{r+1}\left(f, 2^{-k}\right)_{B}^{q} \leq C_{2} \sum_{l=0}^{k}\left[2^{(l-k) r} E_{2^{l}}(f)_{B}\right]^{q} 2^{l-k}
$$

with $C_{2}$ independent of $f$ and $k$. Substituting (3.6) in (3.5), we conclude

$$
\begin{aligned}
\omega^{r}\left(f, 2^{-n}\right)_{B} & \leq C \cdot C_{2} 2^{-n r}\left(\sum_{k=0}^{n} 2^{k r q} \sum_{l=0}^{k} 2^{(l-k) r q} 2^{l-k} E_{2^{l}}(f)_{B}^{q}\right)^{1 / q} \\
& \leq C \cdot C_{2} 2^{-n r}\left(\sum_{l=0}^{n} 2^{l r q} E_{2^{l}}(f)_{B}^{q} \sum_{k=l}^{n} 2^{l-k}\right)^{1 / q} \\
& \leq C_{3} 2^{-n r}\left(\sum_{l=0}^{n} 2^{l r q} E_{2^{l}}(f)_{B}^{q}\right)^{1 / q}
\end{aligned}
$$

which is equivalent to (3.1).

As a corollary of the technique used in the proof of Theorem 3.1, we also obtain the following result.

Theorem 3.2. Suppose $B$ is a Banach space of functions on $\mathbb{T}^{d}$ satisfying $\|f(\cdot)\|_{B}$ $=\|f(\cdot+h)\|_{B},\|f(\cdot+h)-f(\cdot)\|_{B}=o(1)$ for $h \rightarrow 0+$, whenever $f \in B$, and $T_{n} \in B$ for all trigonometric polynomials $T_{n}$. Then

$$
\omega^{r}(f, t)_{B} \leq C t^{r}\left\{\sum_{n \leq \frac{1}{t}} n^{r-1} E_{n}(f)_{B}\right\},
$$

where $\omega^{r}(f, t)_{B}$ and $E_{n}(f)_{B}$ are given by (2.6) and (3.2) respectively.

Proof. We use (3.4), which was proved for $B$ of the present theorem, together with (2.5) with $q=1$ (which is well established for $B$ of the present theorem) to complete the proof of (3.7).

For $\mathbb{R}^{d}$ we have the following result. 
Theorem 3.3. Suppose $B$ is an Orlicz space of functions on $\mathbb{R}^{d}$ given by $M(u)$ of Lemma 2.2 for some $q, 1<q \leq 2$. Then

$$
\omega^{r}(f, t)_{B} \leq C t^{r}\left\{\int_{1}^{1 / t} u^{r q-1} E_{u}(f)_{B}^{q} d u+\|f\|_{B}^{q}\right\}^{1 / q},
$$

where

$$
E_{u}(f)_{B}=\inf \left\{\left\|f-\varphi_{u}\right\|_{B}: \operatorname{supp} \hat{\varphi}_{u}(y) \subset[y:|y| \leq u]\right\},
$$

and where

$$
\hat{f}(y)=\int_{\mathbb{R}^{d}} f(\xi) e^{-2 \pi i y \cdot \xi} d \xi .
$$

Proof. By monotonicity of $\omega^{r}(f, t)_{B}, u^{r q-1}$ and of $E_{u}(f)_{B}^{q}$, we have to prove only

$$
\omega^{r}\left(f, 2^{-n}\right)_{B} \leq C 2^{-n r}\left\{\sum_{l=0}^{n} 2^{l r} E_{2^{l}}(f)_{B}^{q}+\|f\|_{B}^{q}\right\},
$$

which is equivalent to (3.8). Using (3.5) (and observing that here $E_{1}(f)_{B}^{q}$ is replaced by $\|f\|_{B}^{q}$ since $c \neq 0$ does not belong to an Orlicz space on $\mathbb{R}^{d}$ for which $M$ is finite), we have only to establish

$$
\left\|\Delta_{h}^{r} \varphi_{u}\right\|_{B} \leq A h^{r} u^{r}\left\|\varphi_{u}\right\|_{B}
$$

with $A$ independent of $h$ and $u$, and follow the proof of Theorem 3.1. We first show (3.10) for $L_{p}\left(\mathbb{R}^{d}\right), 1 \leq p \leq \infty$ (needed here only for $p=1$ and $p=\infty$ ). Using [Da-Di, 04, Theorem 2.1], we have

$$
\left\|\Delta^{r} \varphi_{u}\right\|_{L_{p}\left(\mathbb{R}^{d}\right)} \leq A u^{2 r}\left\|\varphi_{u}\right\|_{L_{p}\left(\mathbb{R}^{d}\right)},
$$

where $\Delta$ is the Laplacian. We now apply $\mathrm{Di}, 89$, Theorem 6.2 ] to obtain

$$
\left\|\left(\frac{\partial}{\partial \xi}\right)^{r} \varphi_{u}\right\|_{L_{p}\left(\mathbb{R}^{d}\right)} \leq C\left\|\Delta^{r} \varphi_{u}\right\|_{L_{p}\left(\mathbb{R}^{d}\right)}^{1 / 2}\left\|\varphi_{u}\right\|_{L_{p}(\mathbb{R})}^{1 / 2} \leq C_{1} u^{r}\left\|\varphi_{u}\right\|_{L_{p}\left(\mathbb{R}^{d}\right)} .
$$

This implies for $1 \leq p \leq \infty$

$$
\left\|\Delta_{h}^{r} \varphi_{u}\right\|_{L_{p}\left(\mathbb{R}^{d}\right)} \leq C_{2} h^{r} u^{r}\left\|\varphi_{u}\right\|_{L_{p}\left(\mathbb{R}^{d}\right)} .
$$

We use the linear operator $O=\Delta_{h}^{r} \eta_{u}$ with the delayed means operator $\eta_{u}$ (satisfying $\eta_{u} \varphi_{u}=\varphi_{u}$ ) given by

$$
\left(\eta_{u}(f)\right)^{\wedge}(x)=\eta\left(\frac{|x|}{u}\right) \hat{f}(x), \quad u>0,
$$

where $\eta(y) \in C^{\infty}\left(\mathbb{R}^{d}\right), \eta(y)=0$ for $y \leq 1$ and $\eta(y)=0$ for $y \geq 2$ (see Da-Di, 04, Section 2]). We now follow [Ka-Ma, Theorem 1.1] to obtain (3.10) for our Orlicz space. Actually (3.10) can be proved directly and for a wider class of spaces, but as it is just a step in our proof, it will lead too far away from the topic. We now follow the proof of Theorem 3.1 to obtain $(3.8)^{\prime}$.

For the sphere, the analogue of Theorems 3.1 and 3.3 is given in the following result. 
Theorem 3.4. For an Orlicz space $B$ on $S^{d-1}$ and $\omega^{r}(f, t)_{B}$ of Theorem 2.4, we have (3.1), where

$$
E_{n}(f)_{B}=\inf \left\{\left\|f-\varphi_{n}\right\|_{B}: \varphi_{n} \in \operatorname{span} \bigcup_{k<n} H_{k}\right\}
$$

$$
H_{k}=\{\varphi: \widetilde{\Delta} \varphi=-k(k+d-2) \varphi\} \quad \text { and } \quad \widetilde{\Delta} f(x)=\Delta f\left(\frac{x}{|x|}\right) \quad \text { for } x \in S^{d-1} .
$$

Proof. Following the proof of Theorem 3.1] and of [Di, 99, Theorem 4.2], we observe that it is sufficient to prove for our space $B$

$$
\left\|\Delta_{\rho}^{r} \varphi_{n}\right\|_{B} \leq C t^{r} n^{r}\left\|\varphi_{n}\right\|_{B} \quad \text { for } \quad \rho \in O_{t} \quad \text { and } \quad \varphi_{n} \in \operatorname{span} \bigcup_{k<n} H_{k} .
$$

The inequality (3.12) was proved in Di, 99, (4.5)] for $1 \leq p \leq \infty$, and using [Ka-Ma, Theorem 1.1] on the linear operator $O=\Delta_{\rho}^{r} \eta_{1 / t}$ with the delayed mean $\eta_{1 / t}$, to (3.12) for $B=L_{1}\left(S^{d-1}\right)$ and $B=L_{\infty}\left(S^{d-1}\right)$, we obtain (3.12) for our Orlicz space and complete the proof.

\section{REFERENCES}

[Da-Di, 04] F. Dai and Z. Ditzian, Combinations of multivariate averages, J. Approx. Theory, 131 (2004), 268-283. MR2106541

[Da-Di, 05] F. Dai and Z. Ditzian, Littlewood-Paley theory and a sharp Marchaud inequality, Acta Sci. Math. (Szeged), 71 (2005), 65-90. MR2160356 (2006d:26003)

[Di, 80] Z. Ditzian, Some remarks on approximation theorems on various Banach spaces, J. Math. Anal. Appl., 77 (1980), 567-576. MR0593235 (81k:41010)

[Di, 88] Z. Ditzian, On the Marchaud-type inequality, Proc. Amer. Math. Soc., 103 (1988), 198-202. MR0938668 (89d:26004)

[Di, 89] Z. Ditzian, Multivariate Landau-Kolmogorov type inequality, Math. Proc. Cambridge Phil. Soc., 105 (1989), 335-350. MR0974990(90a:26023)

[Di, 99] Z. Ditzian, A modulus of smoothness on the unit sphere, J. Anal. Math., 79 (1999), 189-200. MR.1749311 (2000m:41027)

[Ka-Ma] A. Y. Karlovich and L. Maligranda, On the interpolation constant for Orlicz spaces, Proc. Amer. Math. Soc., 129 (2001), 2727-2739. MR1838797 (2002d:46028)

[Ma-Tr] R. P. Maleev and S. L. Troyanski, On the moduli of convexity and smoothness in Orlicz spaces, Studia Math., 54 (1975), 131-141. MR0388067(52:8904)

[Ti] M. F. Timan, Converse theorem of the constructive theory of functions in the space $L_{p}$, Sb. Math., 44 (88) (1958), 125-132 [in Russian].

[To] V. Totik, Sharp converse theorem for $L^{p}$ polynomial approximation, Constr. Approx., 4 (1988), 419-433. MR0956177 (90k:41015)

[Zy] A. Zygmund, A remark on the integral modulus of continuity, Univ. Nac. Tucumán Rev. Ser. A7 (1950), 259-269. MR0042479(13:118f)

Department of Mathematical and Statistical Sciences, University of Alberta, Edmonton, Alberta, Canada T6G 2G1

E-mail address: zditzian@math.ualberta.ca

Department of Mathematical and Statistical Sciences, University of Alberta, Edmonton, Alberta, Canada T6G 2G1

E-mail address: prymak@gmail.com 